\title{
Stem cells in three-dimensional bioprinting: Future perspectives
}

\author{
Jameel Osborne $\mathrm{MS}^{1}$, Jessica Hellein $\mathrm{BS}^{1}$, Reetish Singla $\mathrm{HS}^{1}$, \\ Pawan K Singal PhD FAHA ${ }^{2}$, Dinender K Singla PhD FAHA FIACS ${ }^{1}$
}

\begin{abstract}
J Osborne, J Hellein, R Singla, PK Singal, DK Singla. Stem cells in three-dimensional bioprinting: Future perspectives. Curr Res Cardiol 2015;2(4):193-196.

Three-dimensional (3D) bioprinting is a new technology used to create biological constructs, widening the scope of regenerative and therapeutic medicine. Stem cells are self-renewing, remain undifferentiated unless stimulated and have the capability to differentiate into all specialized cell types. These characteristics make stem cells ideal for use in the production
\end{abstract}

Cardiovascular disease is a major health burden in the world. Diseases that affect the heart are typically treated using various methods such as pharmaceuticals and surgical procedures to repair damaged tissue and/or provide interventional therapy. Each day, more individuals are being placed on the transplant wait list for new organs. With increased efficiency, patient specificity and customization, threedimensional (3D) printing of biological constructs open a new door for better disease treatment.

Since their discovery, stem cells have become key components in developing new therapies within the field of regenerative medicine. These undifferentiated cells have the capacity to self-renew as well as differentiate into any desired cell type and adapt to the new host environment in the body. The unique potential of stem cells opens a new venue to use these cells in regenerative medicine through the use of $3 \mathrm{D}$ bioprinting.

This bioprinting technology has the capability to print cells with biological support materials that should direct and promote cell growth, and are expected to successfully adapt and integrate into the area of damaged tissue. Most recently, there has been increased interest in stem cell use within the field of bioprinting. Stem cells combined with bioprinting create new possibilities regarding regenerative medicine (1). For example, layered skin tissue and intricate structures, such as a heart valve and vasculature, have been created using this technology (2-4).

The present review explores the different types of stem cells and their corresponding areas of research, discusses the challenges and limitations associated with stem cells, and describes the future perspectives of stem cells in the field of bioprinting.

\section{STEM CELLS}

Stem cells are undifferentiated and self-renewing cells that have the ability to differentiate into the various specialized cell types found in the body. Embryonic stem cells were the first of the three types of stem cells to be successfully isolated. Evans and Kaufman (5) first isolated mouse embryonic stem cells, as described in their 1981 article published in Nature. Since then, the field of stem cell biology has gained significant momentum and excitement.

There are three main types of stem cells: embryonic stem cells (ESCs); induced pluripotent stem cells (iPSCs); and adult stem cells

\begin{abstract}
of 3D printed constructs. A biological construct is composed of cells in a scaffold that is compatible, biomimics and can integrate in vivo. Biological structures generated using $3 \mathrm{D}$ bioprinting have the potential to alleviate the need for donor tissue and organs for transplantation. Additionally, these constructs also provide a better way to model disease and test pharmaceuticals.
\end{abstract}

Key Words: Biological constructs; Organ transplantation; Regenerative medicine; Stem cell therapy

(ASCs). Each of these types of stem cells will be discussed further in their respective sections, along with the associated therapeutic applications.

ESCs

ESCs are isolated from the inner cell mass of the preimplantation blastocyst of an embryo and then maintained in vitro. At this stage of the ESC's life, they are undifferentiated and pluripotent, making them ideal for therapeutic applications (6). Additionally, ESCs are selfrenewing, meaning they divide to make more ESCs while maintaining their undifferentiated state. These cells can be stimulated to differentiate into the varying specialized cell types.

A study by D'Amour et al (7) showed successful differentiation of human ESCs into pancreatic horomone-expressing cells in vitro (7). These cells produced insulin, glucagon, somatostatin, pancreatic polypeptide and ghrelin, all of which are important for pancreatic function. Insulin is of particular importance because diabetic patients lack the ability to readily produce it. The cells produced in this study showed promising treatment potential for patients with diabetes mellitus (7).

In a study by Taiani et al (8), mouse ESCs were differentiated into osteoblasts, suspended in a collagen I gel, and then injected into an osteoporotic mouse model at the site of a burr-hole fracture. The site of the fracture was monitored over a four-week span using microtomography, and it showed great improvement in the diseased bone. This showed promise for potential use of embryonic stem cells in fracture repair and bone regeneration.

Methods to regenerate heart tissue have generated significant interest, as cardiovascular disease continues to lead in the number of mortailities per year. There has been extensive work performed on ESC therapies for cardiovascular disease (9-12). In an early study by one of these groups, Singla et al (12) implanted mouse ESCs into a myocardial infarction mouse model. Postimplantation, these ESCs were shown to differentiate into cardiomyocytes, endothelial cells and vascular smooth muscle cells. Moreover, these cells were also able to successfully migrate into the area of damaged tissue and improve cardiac function. More recently, Singla et al (11) injected mouse ESCs and conditioned media into the damaged heart tissue of mice with doxorubicin-induced cardiomyopathy. The results of this

${ }^{1}$ Division of Metabolic and Cardiovascular Sciences, Burnett School of Biomedical Sciences, College of Medicine, University of Central Florida, Orlando,

Florida, USA; ${ }^{2}$ Institute of Cardiovascular Sciences, St Boniface Research Centre, University of Manitoba, Winnipeg, Manitoba

Correspondence: Dr Dinender K Singla, Division of Metabolic and Cardiovascular Sciences, University of Central Florida, 4110 Libra Drive, Orlando,

Florida 32816, USA. Telephone 407-823-0953, fax 407-823-0956, e-mail dsingla@ucf.edu 
study showed improvement in the fractional shortening and ejection fraction of the heart tissue while also inhibiting apoptosis and promoting vascularization of the tissue. These studies provide promising insight and a foundation for future therapies for cardiac regeneration.

Although their potential is significant, there are limitations associated with this type of stem cell therapy. One major concern associated with ESCs is teratoma (tumour) formation after transplantation $(13,14)$. Another concern is immunological response, which occurs when a foreign object is introduced in the body and is rejected. This rejection of foreign material can potentially lead to death of the recipient. Ethical concerns regarding ESCs are another factor that has plagued this field of research. For years, there has been debate on the legal and moral issues regarding the use of an embryo (13). Many believe that life begins at conception, and the practice of using these cells is unethical. Moreover, some are displeased with government funding being given to research that they do not morally agree with or goes against their religious beliefs. The advent of iPSCs helped to redefine the field of stem cell research and alleviate the ethical concerns (15).

\section{iPSCs}

iPSCs are pluripotent and self-renewing cells that have similar characteristics to ESCs, and are derived from somatic cells through transfection of transcription factors using viral or nonviral methods (16). iPSCs are created by taking adult somatic cells from the body, and reprogramming them using four factors, Oct 3/4, Sox 2, Klf 4 and c-Myc $(16,17)$. These factors genetically reprogram the cell from a specialized state to one that is ESC-like. The transformed cells now have the ability to differentiate into cell types found in all three germ layers: ectoderm, endoderm and mesoderm. This is useful because all specialized cell types found in the body are derived from one of these layers. When examining their growth, iPSCs were positive for embryonic cells markers, such as Nanog and SSEA-3, and also had a morphology and cell staining comparable with that of ESCs (17). These cells have eliminated ethical concerns associated with ESCs because embryos are not being used, making iPSCs even more desirable candidates for therapeutic and regenerative applications.

The process of transforming somatic cells into iPSCs was first performed on adult mice somatic cells in 2006 (17). Members of the same group (Takahashi et al [17]) later showed that human fibroblasts taken from the human dermis could be converted into iPSCs, using the same four transcription factors, and that the generated iPSCs exhibit the same characteristics as human ESCs. Fibroblasts and adipose tissue are commonly used to create iPSCs; however, somatic cells can be taken from anywhere in the body. Singla et al (18) showed the first successful transformation of H9C2 cells, also known as cardiomyoblasts, into iPSCs using the transcription factors mentioned previously. These cells were then differentiated into cardiomyocytes. This study showed that regeneration of heart tissue after a myocardial infarction was possible using cardiomyocytes differentiated from iPSC (18).

iPSCs were created to circumvent the ethical concerns associated with ESCs. Another major benefit associated with iPSCs include their ability to be generated directly from a patient population. This enables the creation of patient-specific therapies while simultaneously eliminating the concern of immunological response (16). Moreover, because these cells are derived from patient somatic cells, the supply is more plentiful compared with some other type of stem cells, such as ASCs. Concerns associated with iPSCs are the method of synthesis is inefficient, and viral methods for transformation may incorporate into the genome of the cell causing harm and teratoma formation (16).

\section{ASCs}

ASCs reside in the organs and tissue of the body that remain in an undifferentiated state and are self-renewing (19). When external stimuli prompt ASCs, such as the time when the body needs to heal or regenerate, they are able to differentiate into the specialized cell types of the tissue in which they are found. ASCs are found in most tissues, although some have higher quantities than others. For example, hematopoietic stem cells (HSCs), which are found in the bone marrow and generate all of the components of blood, exist in higher quantities compared with cardiac and neural stem cells (19).

ASCs have been used for therapies for a relatively long time. Specifically, HSCs have been used for many decades for transplantation to treat blood-related diseases (19). It was originally believed that HSCs were limited in their multipotency. ASCs initially were believed to be limited in plasticity, meaning that they were only able to differentiate into the specialized cells in the tissue in which they were found; however, recent research has shown greater differentiation capabilities (20). A study by Tang et al (20) showed that musclederived stem cells grown in Schwann cell culture with factors PDGF, NT-3 and IGF-2 would transdifferentiate into neural cells. Moreover, adipose ASCs have been shown to be able to differentiate into other cell types, such as hepatocytes (21). While this is an enormous feat to be able to differentiate an ASC to a cell of a completely different lineage, there remains issues with the approach.

One issue is that ASC-derived cells do not naturally express all of the tissue-specific molecules and markers usually present on a native cell (21). For example, it has been shown that adipose cells, differentiated into hepatocytes, did not express five common hepatocyte factors, thus affecting the ability of the transformed ASCs to function correctly (21). Missing key components such as those will undoubtedly affect the efficacy and function of a cell.

Isolating and obtaining ASCs can be difficult, time consuming and laborious, especially for tissue with a small ASC reservoir. While ASCs do have the ability to transdifferentiate, their plasticity remains limited compared with that of the other two types of stem cells. Similar to iPSCs, however, the immune response is not an issue, if the ASCs are patient derived and specific.

\section{LIMITATIONS OF THERAPEUTIC STEM CELL USE}

As described in their respective sections, each type of stem cell has limitations. In summary, ESCs are limited primarily by ethical concerns, immune response and teratoma formation. iPSCs also exhibit teratoma formation like in ESCs, are often inefficiently produced, and the viral method of transfection can potentially harm the cells by incorporating into the cell's genome. Finally, ASCs are limited in plasticity; furthermore, they are difficult to obtain and are found in limited quantities.

While each type of stem cell has its own advantages as well as limitations, there are some limitations that affect all types of cells regarding in vivo use for regeneration. Engraftment and integration of cells into the recipient's natural environment of the body has shown to be a major challenge (14). Cellular integration difficulties can hinder the improvement of damaged tissue in the body and, therefore, give rise for the need of further and more refined strategies. The major, and relatively new, strategy addressed in the present review is the concept of $3 \mathrm{D}$ printing of biological constructs, to successfully overcome the aforementioned challenges.

\section{D BIOPRINTING}

Bioprinting, a form of tissue engineering, is an emerging discipline within the fields of regenerative and therapeutic medicine $(22,23)$. The practice of bioprinting itself is multidisciplinary in nature, encompassing the fields of biology, materials science and engineering. The central dogma of tissue engineering is obtaining cells, placing the cells on a biologically compatible scaffold, and maintaining the conditions necessary for cell survival and proliferation while also introducing extracellular signals necessary to direct cells toward tissue formation (Figure 1). With bioprinting being a relatively new field, there is still much to discover and learn before this type of technology can be streamlined for medical use $(22,23)$. This section will outline the basic functionality of the various types of $3 \mathrm{D}$ bioprinters, what factors to consider when designing and printing a biological construct, the current research regarding $3 \mathrm{D}$ bioprinting and the future perspectives of this field. 


\section{Types of printers}

3D bioprinting began with the transformation of a commercial desktop inkjet printer to the one that can print biological material (24). Inkjet bioprinting is one of the various types of bioprinters currently being used in research. This type of print technology is modified by substituting the cartridge ink with cells and cellular material, modifying the print head to give the appropriate print droplet size, deposition, consistency and uniformity and, finally, modifying the software for use in biological application (23). The cells are deposited in a layer-by-layer fashion (23), much like the movements of a normal twodimensional desktop printer, while incorporating a z-axis, which allows the print stage to move up and down (ie, in the third dimension). While inkjet is the most commonly used type of bioprinting, microextrusion and laser-assisted bioprinters are also being used.

Microextrusion bioprinters use either pressure or mechanical methods to dispense the cellular material in programmed locations (23). Microextrusion printers in general are fairly common. Nonbiological microextrusion printers encompass many types of commonly used 3D plastic printers. One benefit of this type of printer is that it already is designed with three axes, unlike the inkjet, in which the addition of the third axis is a necessary modification. This printing technology works by depositing the material in the specified locations by fluid motions of the extruder (23). A wide variety of materials can be used with this printer, such as plastics, hydrogels and copolymers, making it fairly versatile (23).

The third type, laser-assisted bioprinters, uses a significantly different technique than the previous two printers. This type of bioprinter uses a laser pulse to push the biological material to specific areas on a collector region $(23,25)$. This technique is the most complex, and also the most expensive of the three options. This type of printer is not as commonly used as inkjet and microextrusion; however, it does show promise in the realm of bioprinting, and is gaining interest among researchers because it is shown to produce prints with high viability and resolution (25).

\section{MATERIAL FACTORS}

Cells, biologically compatible scaffolding and extracellular factors are the three main components needed to build a biological construct. With stem cells having been discussed in a greater detail in the previous section, this section focuses on scaffold design and culturing of $3 \mathrm{D}$ printed tissue.

Stem cells have great potential for regenerative therapies; however, one main issue with the use of stem cell therapies currently is integration and engraftment of cells into the body. If these cells are not able to integrate and biomimic the natural environment properly in vivo, regeneration of the tissue cannot be fully accomplished, and also has the potential to cause additional issues (23). Materials play a significant role in cell attachment and proper cellular function (23). These scaffolds must be able to allow the cells to successfully integrate and proliferate within them, while also not harming the cells in the process. Moreover, these constructs must also cause no harm to the in vivo tissue (23). Careful consideration and research must be performed to determine whether implanting these engineered structures will cause unwanted adverse effects.

When building a biological construct, many factors must be taken into account. These include, but are not limited to, biocompatibility, ability to biologically mimic the in vivo environment, nontoxicity, integration, and mechanical and chemical properties of the materials (23). Cells must be able to integrate, proliferate, maintain viability and function the same as a natural cell in the body. Scaffolds essentially act as the extracellular matrix of the cell (23).

One design strategy when making a scaffold is to have the construct stay in vivo. The other, and arguably better, option is to make a scaffold that degrades over time. If the construct is going to stay in vivo, it must be made of materials that will not harm the body for long periods of time, and is designed to accommodate the dynamic nature of the extracellular matrix and cell (23). A degradable scaffold and

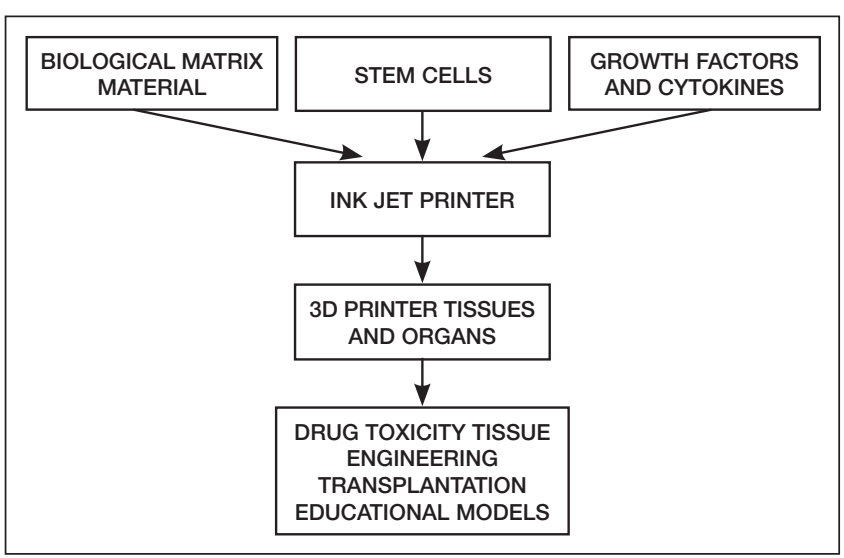

Figure 1) Schematic representation of three-dimensional (3D) printing, organ development and applications

its byproducts must also be nontoxic and noncarcinogenic (23). The process of designing a scaffold is a delicate balance of finding what will allow the cells to adhere, proliferate and differentiate, while also ensuring that no materials will harm the cells and in vivo environment.

\section{TISSUE ENGINEERING USING 3D BIOPRINTING}

Once bioprinting is established, the use of stem cells as the cellular material of choice will gain interest in the scientific community (1). Stem cells' pluripotency and self-renewal make them appealing cellular material for use in 3D bioprinting.

Bioprinting began with a thermal inkjet printer. Boland et al (26) and others (27-29), performed the preliminary work in the field using a modified Hewlett Packard 550 inkjet printer, and patented this inkjet printing technology in 2006 (24,26-29). In one study by this group, the viability of Chinese hamster ovary cells and rat embryonic motoneurons was examined after they were printed, and this group showed cell survival to be $>90 \%$ post-printing (28). A study by Cui and Boland (2) showed that human vasculature could be created by printing human microvascular endothelial cells with fibrinin. This creation is significant because vasculature is necessary for 3D printed structures to survive in vivo, because the tissue will need blood supply (2). While these studies did not use stem cells, they laid the foundation for the field of stem cell bioprinting.

While bioprinting with stem cells is a newer practice, there have been many tissues printed already. In a study by Gao et al (30), human mescenchymal stem cells were printed using inkjet technology with a PEG-GelMA scaffold, and created tissue, with $>80 \%$ of the cells surviving post-printing. In a recent study by Hsieh et al (31), mild improvement of the central nervous system of zebrafish was observed when injected with 3D printed neural stem cells. In 2013, Xu et al (32) demonstrated the ability to print multiple cell types simultaneously. In this study, human amniotic-derived stem cells, canine smooth muscle cells and bovine aortic endothelial cells were each mixed with $\mathrm{CaCl}_{2}$ and printed, using thermal inkjet technology, into an alginate-collagen scaffolding. In vitro results showed the tissue maintained the cell-specific characteristics of each printed cell type. In vivo, all cell types survived engraftment, and showed vascularization of the tissue (32). Engraftment and vascularization are essential for a 3D bioprinted construct to survive in vivo.

While inkjet bioprinting is the most common approach to bioprinting to date, there has been successful printing of stem cells using other methods. As shown in the study by Gaebel et al (33), cardiac patches for in vivo therapeutics were created and seeded with human umbilical vein endothelial cells and human mesenchymal stem cells by laser-induced forward transfer cell printing. When the patch seeded with the cells was placed in vivo, the results showed that there was improvement in function as well as an increase in the vasculature of the 
region damaged by myocardial infarction (33). Moreover, Ali et al (25) showed that mesenchymal stem cells printed using laser-assisted printing had high viability, reportedly almost $100 \%$, as well as high resolution postprint. High viability and resolution are also essential for a successful $3 \mathrm{D}$ printed product.

\section{FUTURE PERSPECTIVES}

Although 3D bioprinting is currently in it early stages, the outlook for this field is tremendous. In developed countries, chronic diseases lead in number of mortalities per year by far compared with infectious diseases. The ability to print tissues and organs provides novel therapeutic opportunities for these kinds of diseases that cannot solely be accomplished with medication, lifestyle changes or surgical procedures. As described previously, many biological constructs have already been successfully printed. One of many examples can be seen in the study by Duan et al (3), which details the production of a successful 3D trileaflet valve. This type of valve could be used as a replacement for damaged or diseased valves.

Standardization and continued improvement of printed biological constructs are needed before clinical use becomes common. Future

\section{REFERENCES}

1. Tasoglu S, Demirci U. Review: Bioprinting for stem cell research. Trends Biotechnol 2013;31:10-9.

2. Cui $X$, Boland T. Human microvasculature fabrication using thermal inkjet printing technology. Biometerials 2009;30:6221-7.

3. Duan B, Kapetanovic E, Hockaday L, Butcher J. Three-dimensional printed trileaflet valve conduits using biological hydrogels and human valve interstitial cells. Acta Biomaterialia 2014;10:1836-46.

4. Lee V, Singh G, Karande P, et al. Design and fabrication of human skin by three-dimensional bioprinting. Tissue Engineering Part C-Methods 2014;20:473-84.

5. Evans M, Kaufman M. Establishment in culture of pluripotential cells from mouse embryos. Nature 1981;292:154.

6. Solter D. From teratocarcinomas to embryonic stem cells and beyond: A history of embryonic stem cell research. Nat Rev Genet 2006;7:319-27.

7. D'Amour K, Bang A, Baetge E, et al. Production of pancreatic hormone-expressing endocrine cells from human embryonic stem cells. Nat Biotechnol 2006;24:1392-401.

8. Taiani J, Buie H, Matyas J, et al. Embryonic stem cell therapy improves bone quality in a model of impaired fracture healing in the mouse; tracked temporally using in vivo micro-CT. Bone 2014;64:263-72.

9. Kumar D, Kamp T, LeWinter M. Embryonic stem cells: Differentiation into cardiomyocytes and potential for heart repair and regeneration. Coronary Artery Dis 2005;16:111-6.

10. Kumar D, Sun B. Transforming growth factor- $\beta 2$ enhances differentiation of cardiac myocytes from embryonic stem cells. Biochem Biophys Res Commun 2005;332:135-41.

11. Singla D, Abdelli L. Embryonic stem cells and released factors stimulate c-kit (+)/FLK-1(+) progenitor cells and promote neovascularization in doxorubicin-induced cardiomyopathy. Cell Transplant 2015;24:1043-52.

12. Singla D, Hacker T, Kamp T, et al. Transplantation of embryonic stem cells into the infarcted mouse heart: Formation of multiple cell types. J Mol Cell Cardiol 2006;40:195-200.

13. Mountford J. Human embryonic stem cells: Origins, characteristics and potential for regenerative therapy. Transfusion Med 2008;18:1-12

14. Singla D. Embryonic stem cells in cardiac repair and regeneration. Antioxid Redox Signal 2009;11:1857-63.

15. De Lázaro I, Yilmazer A, Kostarelos K. Induced pluripotent stem (iPS) cells: A new source for cell-based therapeutics? J Control Release 2014;185:37-44.

16. Stadtfeld M, Hochedlinger K. Induced pluripotency: History, mechanisms, and applications. Genes Develop 2010;24:2239-63.

17. Takahashi K, Yamanaka S. Induction of pluripotent stem cells from mouse embryonic and adult fibroblast cultures by defined factors. Cell 2006;126:663-76.

18. Singla DK, Long X, Glass C, Singla RD, Yan B. Induced pluripotent stem (iPS) cells repair and regenerate infarcted myocardium. Mol Pharm 2011;8:1573-81. bioprinting applications can include, but are not limited to, production of tissue for wound repair, models for drug toxicity studies, pharmaceutical testing and, eventually, organs for transplantation $(1,23)$. While standardizing practices to create therapies using 3D biological constructs may be not be attainable at the current moment, further advances in this field will undoubtedly revolutionize current research techniques and provide novel treatments for disease.

ACKNOWLEDGEMENTS: Dr Pawan K. Singal is the holder of the Dr Naranjan S Dhalla Chair in Cardiovascular Research supported by the St Boniface Hospital and Research Foundation. The authors are very thankful for the part of the support provided by The Estate of Rebecca Gurecki.

DISCLOSURES: The authors have no financial disclosures or conflicts of interest to declare.

19. Sousa B, Parreira R, Resende R, et al. Human adult stem cells from diverse origins: An overview from multiparametric immunophenotyping to clinical applications. Cytometry Part A J Int Socr Analytical Cytol 2014;85:43-77.

20. Tang Y, He H, Jiang H, et al. PDGF, NT-3 and IGF-2 in combination induced transdifferentiation of muscle-derived stem cells into Schwann cell-like cells. PLoS One 2014;9:1-7.

21. Lue J, Lin G, Ning H, Xiong A, Lin C, Glenn J. Transdifferentiation of adipose-derived stem cells into hepatocytes: A new approach. Liver Int 2010;30:913-22.

22. Mironov V, Boland T, Trusk T, Forgacs G, Markwald R. Organ printing: Computer-aided jet-based 3D tissue engineering. Trends Biotechnol 2003;21:157-61.

23. Murphy S, Atala A. 3D bioprinting of tissues and organs. Nat Biotechnol 2014;32:773-85.

24. Xu T, Jin J, Gregory C, Hickman J, Boland T. Inkjet printing of viable mammalian cells. Biomaterials 2005;26:93-9.

25. Ali M, Pages E, Ducom A, Fontaine A, Guillemot F. Controlling laser-induced jet formation for bioprinting mesenchymal stem cells with high viability and high resolution. Biofabrication 2014;6:045001

26. Boland T, Tao X, Bhaduri S, et al. Drop-on-demand printing of cells and materials for designer tissue constructs. Mat Sci Eng C 2007;27:372-6

27. Cui X, Boland T, D'Lima D, Lotz M. Thermal inkjet printing in tissue engineering and regenerative medicine. Recent Pat Drug Deliv Formul 2012;6:149-55.

28. Cui X, Dean D, Ruggeri Z, Boland T. Cell damage evaluation of thermal inkjet printed Chinese hamster ovary cells. Biotechnol Bioengineer 2010;106:963-9.

29. Xu T, Gregory C, Boland T, et al. Viability and electrophysiology of neural cell structures generated by the inkjet printing method. Biomaterials 2006;27:3580-8.

30. Gao G, Schilling A, Cui X, et al. Improved properties of bone and cartilage tissue from 3D inkjet-bioprinted human mesenchymal stem cells by simultaneous deposition and photocrosslinking in PEG-GelMA. Biotechnol Lett 2015;37:2349.

31. Hsieh F, Lin H, Hsu S. 3D bioprinting of neural stem cell-laden thermoresponsive biodegradable polyurethane hydrogel and potential in central nervous system repair. Biomaterials 2015;71:48-57.

32. Xu T, Zhao W, Zhu J, Albanna M, Yoo J, Atala A. Complex heterogeneous tissue constructs containing multiple cell types prepared by inkjet printing technology. Biomaterials 2013;34:130-9.

33. Gaebel R, Ma N, Steinhoff G, et al. Patterning human stem cells and endothelial cells with laser printing for cardiac regeneration. Biomaterials 2011;32:9218-30. 